\title{
$\gamma$ Pegasi: testing Vega-like magnetic fields in B stars ${ }^{\star \star \star}$
}

\author{
C. Neiner ${ }^{1}$, D. Monin ${ }^{2}$, B. Leroy ${ }^{1}$, S. Mathis ${ }^{3,1}$, and D. Bohlender ${ }^{2}$ \\ 1 LESIA, Observatoire de Paris, CNRS UMR 8109, UPMC, Université Paris Diderot, 5 place Jules Janssen, 92190 Meudon, France \\ e-mail: coralie.neiner@obspm.fr \\ 2 Dominion Astrophysical Observatory, Herzberg Astronomy and Astrophysics Program, National Research Council of Canada, \\ 5071 West Saanich Road, Victoria, BC V9E 2E7, Canada \\ ${ }^{3}$ Laboratoire AIM Paris-Saclay, CEA/DSM-CNRS-Université Paris Diderot, IRFU/SAp, Centre de Saclay, \\ 91191 Gif-sur-Yvette Cedex, France
}

Received 20 November 2013 / Accepted 13 December 2013

\section{ABSTRACT}

\begin{abstract}
Context. The bright B pulsator $\gamma$ Peg shows both $\mathrm{p}$ and $\mathrm{g}$ modes of $\beta$ Cep and SPB types. It has also been claimed that it is a magnetic star, while others do not detect any magnetic field.

Aims. We check for the presence of a magnetic field, with the aim to characterise it if it exists, or else provide a firm upper limit of its strength if it is not detected. If $\gamma$ Peg is magnetic as claimed, it would make an ideal asteroseismic target for testing various theoretical scenarios. If it is very weakly magnetic, it would be the first observation of an extension of Vega-like fields to early B stars. Finally, if it is not magnetic and we can provide a very low upper limit on its non-detected field, it would make an important result for stellar evolution models.

Methods. We acquired high resolution, high signal-to-noise spectropolarimetric Narval data at Telescope Bernard Lyot (TBL). We also gathered existing dimaPol spectropolarimetric data from the Dominion Astrophysical Observatory (DAO) and Musicos spectropolarimetric data from TBL. We analysed the Narval and Musicos observations using the least-squares deconvolution (LSD) technique to derive the longitudinal magnetic field and Zeeman signatures in lines. The longitudinal field strength was also extracted from the $\mathrm{H} \beta$ line observed with the DAO. With a Monte Carlo simulation we derived the maximum strength of the field possibly hosted by $\gamma$ Peg.

Results. We find that no magnetic signatures are visible in the very high quality spectropolarimetric data. The average longitudinal field measured in the Narval data is $B_{l}=-0.1 \pm 0.4 \mathrm{G}$. We derive a very strict upper limit of the dipolar field strength of $B_{\mathrm{pol}} \sim 40 \mathrm{G}$. Conclusions. We conclude that $\gamma$ Peg is not magnetic: it hosts neither a strong stable fossil field as observed in a fraction of massive stars nor a very weak Vega-like field. There is therefore no evidence that Vega-like fields exist in B stars, contrary to the predictions by fossil field dichotomy scenarios. These scenarios should thus be revised. Our results also provide strong constraints for stellar evolution models.
\end{abstract}

Key words. stars: magnetic field - stars: early-type - stars: individual: $\gamma$ Pegasi

\section{Introduction}

The very bright $(V=2.83)$ B2IV star $\gamma$ Peg hosts both $\mathrm{p}$ and g pulsation modes. From space-based MOST observations, Handler et al. (2009) detected eight $\beta$ Cep-like p-modes and six SPB-like g-modes, with frequencies ranging from 0.6 to $9.1 \mathrm{c} \mathrm{d}^{-1}$. Walczak \& Daszyńska-Daszkiewicz (2010) provide the most probable identification for these modes. In addition, $\gamma$ Peg is an intrinsically very slow rotator, with vsini close to $0 \mathrm{~km} \mathrm{~s}^{-1}$ (Telting et al. 2006) and $v \sim 3 \mathrm{~km} \mathrm{~s}^{-1}$ (Handler et al. 2009). Moreover, $\gamma$ Peg has been claimed to be a multiple star (Chapellier et al. 2006), but McAlister et al. (1989) and Roberts et al. (2007) did not detect any companion. Handler et al. (2009) propose that the observed variations are rather due to the pulsations and that $\gamma$ Peg is a single star.

Finally, Butkovskaya \& Plachinda (2007, hereafter BP07) claimed to have detected a magnetic field in $\gamma$ Peg with a longitudinal field varying from -10 to $30 \mathrm{G}$ with a period

\footnotetext{
* Based on observations obtained at the Telescope Bernard Lyot (USR5026) operated by the Observatoire Midi-Pyrénées, Université de Toulouse (Paul Sabatier), Centre National de la Recherche Scientifique of France, and at the Dominion Astrophysical Observatory.

$\star \star$ Tables 1-3 are available in electronic form at

http://www . aanda.org
}

$P=6.6538 \pm 0.0016 \mathrm{~d}$. This longitudinal field would correspond to an oblique dipole field with a polar field strength of $B_{\mathrm{pol}}=570 \mathrm{G}$, an inclination angle $i=9^{\circ}$ and an obliquity angle $\beta=85^{\circ}$. Silvester et al. (2009), however, detected no magnetic signature in an ESPaDOnS measurement and derived a null longitudinal field value of $B_{l}=6 \pm 11 \mathrm{G}$. Schnerr et al. (2008) also found no magnetic signature in two Musicos measurements with $B_{l}=3 \pm 20 \mathrm{G}$ and $B_{l}=-1 \pm 17 \mathrm{G}$. In addition, Morel et al. (2008) studied the chemical abundances of magnetic pulsating B stars, particularly their N-enrichment, and found that $\gamma \mathrm{Peg}$ stands out as a normal $N$ star, whereas other magnetic pulsating B stars seem to have N-enrichment. Moreover, $\gamma$ Peg appears as non-variable in the UV wind resonance lines, unlike most magnetic massive stars (Schnerr et al. 2008). Therefore doubt can be cast on the detection of any magnetic field in $\gamma$ Peg by BP07.

Whether $\gamma$ Peg hosts a magnetic field is important for several reasons. First of all, Aurière et al. (2007) proposed that dipolar magnetic fields can only exist above a certain polar field strength threshold. They obtained a plateau at $\sim 1000 \mathrm{G}$ and a threshold at $\sim 300 \mathrm{G}$. This critical field value would be necessary for the stability of large-scale magnetic fields. If the field suggested by BP07 existed, it would fall in this category. The nondetection of a magnetic field in $\gamma$ Peg by other authors, however, suggests that if $\gamma \mathrm{Peg}$ is magnetic, its field might be very weak. 
Lignières et al. (2009) discovered a field well below the critical field limit with $B_{l}=-0.6 \pm 0.3 \mathrm{G}$ in the A star Vega (also see Petit et al. 2010) and Petit et al. (2011) discovered a field with $B_{l}=0.2 \pm 0.1 \mathrm{G}$ in the Am star Sirius. These authors suggest that a new class of very weakly magnetic stars may exist among intermediate-mass and massive stars. A dichotomy would then exist between these very weakly magnetic stars, for which the longitudinal field is in the sub-Gauss regime, and the dipolar magnetic stars with field strength above $\sim 300 \mathrm{G}$ at the poles. It is thus interesting to check whether $\gamma$ Peg could be a B counterpart of this new "Vega-like" category.

Second, the presence of a magnetic field in massive stars modifies significantly their evolution. In particular the interaction between rotation and a magnetic field may completely modify the transport of angular momentum and of chemical elements. If the field suggested by BP07 exists, it would inhibit mixing in $\gamma$ Peg (e.g. Spruit 1999; Mathis \& Zahn 2005; Zahn 2011; Briquet et al. 2012). If $\gamma$ Peg hosts a very weak field, its impact on transport will probably depend on the nature of the field, its strength and complexity inside the star. The effect of such weak fields would have to be investigated theoretically (see the discussion in Zahn et al. 2007). Therefore, it is necessary to know whether very weak fields are present in upper main sequence stars and should be included in stellar evolution codes (e.g. Maeder \& Meynet 2003; Heger et al. 2005).

In this paper, we investigate new Narval measurements of the magnetic field in $\gamma$ Peg as well as unpublished archival Musicos and DAO data (Sect. 2). We measure the longitudinal field values with the $\mathrm{H} \beta$ line in the DAO data and with the least-squares deconvolution (LSD) technique in the Musicos and Narval data (Sect. 3). We then derive the upper limit of the non-detected field (Sect. 3.3) and discuss the impact on this non-detection at a very low field level on fossil field theories and evolution models (Sect. 4).

\section{Observations}

\subsection{Musicos observations}

Musicos is a fibre-fed echelle spectropolarimeter with a resolving power of 35000 , which was attached to the 2-m Télescope Bernard Lyot (TBL) at the Pic du Midi in France until 2006 (see Donati et al. 1999). The spectrograph covers the wavelength domain from 4490 to $6619 \AA$.

We collected 34 Stokes $V$ (circular polarisation) measurements of $\gamma$ Peg with Musicos between 2001 and 2005. Their signal-to-noise ratio (S/N) varies between 408 and 1202 at $5000 \AA$ in the I spectrum. See Table 1 .

One Stokes $Q+U$ observation was also obtained on October 9, 2003. However, we consider here only measurements of circular polarisation. Indeed the signatures detected in linear polarisation due to a stellar magnetic field in massive stars is usually 10 to 100 times weaker than the one observed in circular polarisation.

Data were reduced with our own version of the ESPRIT reduction package (Donati et al. 1997). The usual bias and flatfield corrections were applied, as well as a wavelength calibration with a ThAr lamp. Each echelle order was then carefully normalised with the continuum package of the IRAF software ${ }^{1}$.

\footnotetext{
1 IRAF is distributed by the National Optical Astronomy Observatory, which is operated by the Association of Universities for Research in Astronomy (AURA) under cooperative agreement with the National Science Foundation.
}

\subsection{Narval observations}

Narval is a fibre-fed echelle spectropolarimeter with a resolving power of 65000, which replaced Musicos at the TBL in 2006. The spectrograph covers the wavelength domain from 3694 to $10483 \AA$.

We collected 23 Stokes $V$ measurements of $\gamma \mathrm{Peg}$ with Narval in November-December 2007. Their S/N varies between 661 and 1682 at $5000 \AA$ in the I spectrum. See Table 2. Nine of the spectra are saturated in certain wavelength regions. These regions have been rejected.

Data were reduced with the LIBRE-ESPRIT reduction package, an extension of ESPRIT (Donati et al. 1997) for Narval available at the telescope, in a manner similar to that used for the Musicos data.

\subsection{DAO observations}

The dimaPol spectropolarimeter on the 1.8-m DAO Plaskett telescope was used to obtain magnetic field measurements in the hydrogen line $\mathrm{H} \beta$. The spectropolarimeter has a resolving power of 10000 . Spectra in opposite circular polarisations, of approximately $250 \AA$ wide and centred on $\mathrm{H} \beta$, are recorded on the CCD. A fast switching liquid crystal wave plate quickly interchanges the spectra on the detector. Fast switching of the plate combined with charge shuffling on the CCD significantly reduces instrumental effects and increases the accuracy of spectropolarimetric measurements. Details about the instrument and data reduction can be found in Monin et al. (2012).

We collected 18 measurements of $\gamma$ Peg with dimaPol at the DAO between 2007 and 2010. A single observation of $\gamma$ Peg consists of between 20 and 100 sub-exposures of 18 to $60 \mathrm{~s}$ long. Sixty switches per sub-exposure are typically performed. Some observations obtained in 2007 and 2008 were obtained with 2 to 30 switches per sub-exposure. The $\mathrm{S} / \mathrm{N}$ of the measurements varies between 1000 and 2400 at $5000 \AA$ in the I spectrum. See Table 3.

\section{Spectropolarimetric analysis}

\section{1. $H \beta$ analysis of $D A O$ data}

The longitudinal field values $B_{l}$ extracted from DAO observations have been obtained by measuring the Zeeman shift between the two opposite circular polarisations in the core of the $\mathrm{H} \beta$ line with the Fourier cross-correlation technique. This shift is proportional to the longitudinal field (see e.g. Landstreet 1992), with a coefficient of $6.8 \mathrm{kG}$ per pixel for $\mathrm{H} \beta$ for dimaPol. The shift is measured using unnormalised spectra that have not been wavelength calibrated (i.e. in pixel space). We only apply a chromatic correction so that the continuum shape is similar in both polarisations. The window of the Fourier cross-correlation has been adjusted to $2.4 \AA$ in order to include the part of the line profile most sensitive to the magnetic field. See more details about this technique in Monin et al. (2012).

We find that the $B_{l}$ values are all compatible with 0 within $3 \sigma_{B_{l}}$. The field values and their error bars are reported in Table 3. The mean longitudinal field obtained from the $18 \mathrm{DAO}$ measurements is $B_{l}=8 \pm 14 \mathrm{G}$.

In the top panel of Fig. 1 the longitudinal field values obtained from the $\mathrm{H} \beta$ line of DAO observations are shown folded with $P=6.6538 \mathrm{~d}$ and $\mathrm{HJD}_{0}=2450679.364$, the ephemeris suggested by BP07. A sinusoidal dipole fit of the $B_{l}$ data is performed and compared to a null field. While we cannot reproduce 


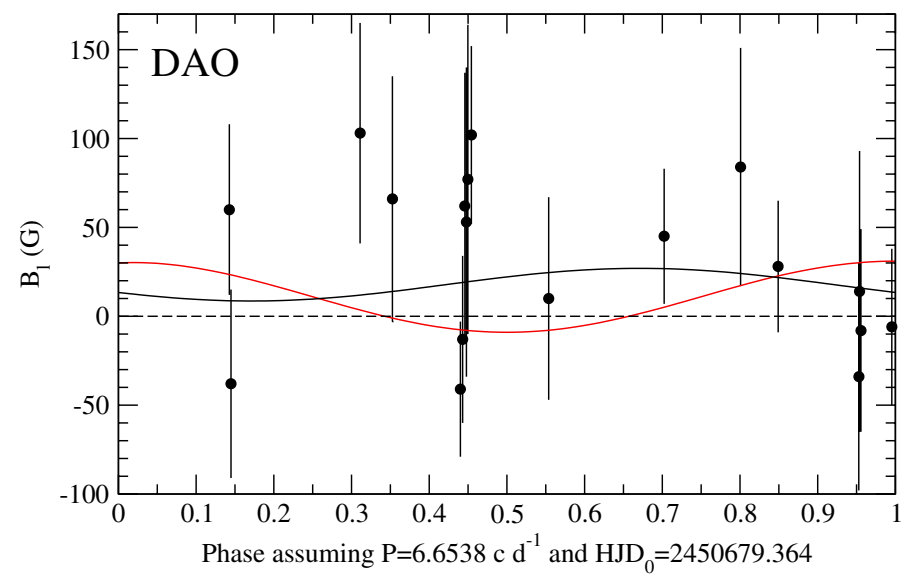

Fig. 1. Longitudinal field measurements from the $\mathrm{H} \beta$ line observed with DAO. The black solid line shows the best sinusoidal dipole fit to the data, the dashed line shows a null field, and the red solid line shows the sinusoidal variation expected from BP07.

the sinusoidal variation expected from BP07 (red line), a dipole fit with their period but different phasing is possible (black line). The best fit is a sinusoid centred at $B_{0}=22.5 \mathrm{G}$ with an amplitude $B=13.2 \mathrm{G}$. It results in a reduced $\chi^{2}=0.86$, while the null field (dashed line) has $\chi^{2}=0.99$. However, the DAO datapoints appear very scattered around this fit. Moreover, considering the distribution of the small number of datapoints over several years, the periodicity possibly present in these data is very badly constrained. Therefore, we are also able to find similarly good sinusoidal fits with a variety of other periods: the Fourier spectrum of the DAO longitudinal field measurement is shown in Fig. 2. As a consequence, the fit of the DAO data with BP07's period does not appear to be significant.

\subsection{LSD analysis of Musicos and Narval data}

We applied the LSD technique (Donati et al. 1997) to the Musicos and Narval data. We first constructed a line mask based on a line template derived from the VALD database (Piskunov et al. 1995; Kupka et al. 1999) with $T_{\text {eff }}=22000 \mathrm{~K}$ and $\log g=3.5$ dex. The template only contains lines with a depth above $1 \%$ of the continuum level. From the line template we removed all hydrogen lines, lines that are blended with $\mathrm{H}$ lines or interstellar bands, as well as lines that did not seem to be present in the Narval spectra. We then adjusted the strength of the remaining lines to fit the Narval observations. This resulted in masks containing 491 lines for Musicos and between 744 and 1012 lines for Narval. There are less lines in Musicos spectra because the wavelength range is smaller. The number of lines for Narval depends on whether we had to discard parts of the wavelength range where the signal was saturated. This number is indicated in Table 2.

Using these line masks, we extracted LSD Stokes $I$ and $V$ profiles for each spectropolarimetric measurements. We also extracted null $(N)$ polarisation profiles to check for spurious signatures, e.g. from instrumental origin or from stellar pulsations.

Figure 3 shows the Narval LSD I profiles folded with the ephemeris proposed by BP07 and with the main pulsation pe$\operatorname{riod}\left(P_{\text {puls }}=0.15175 \mathrm{~d}\right)$ found by Handler et al. (2009). We find that the LSD I profiles indeed vary with the published main pulsation period, but no coherent variation is found with the period published by BP07.

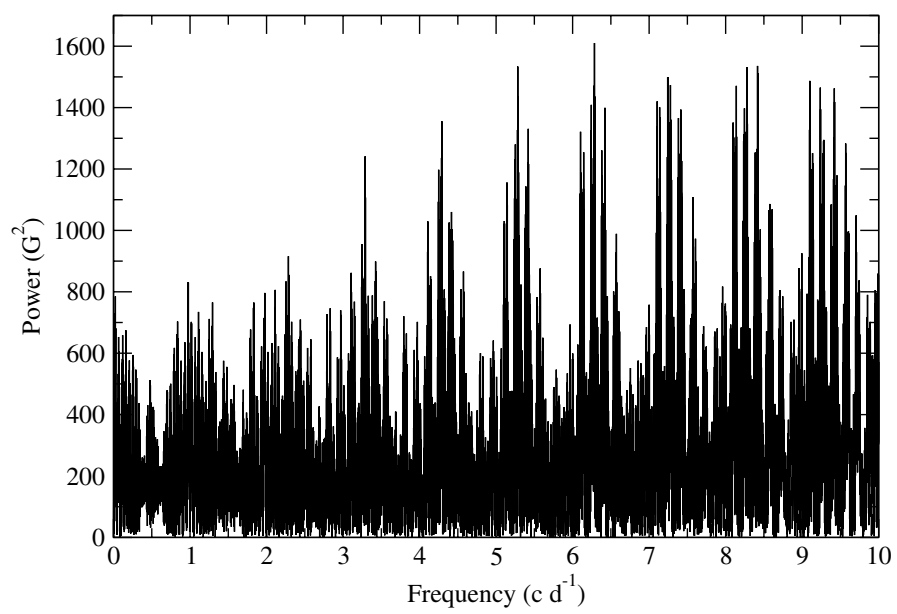

Fig. 2. Power spectrum of the DAO longitudinal field measurements.

We find that all $N$ profiles are flat, i.e. that the measurements have not been polluted, and that all Stokes $V$ profiles are also flat, i.e. we do not detect a magnetic signature in any of the Musicos and Narval measurements.

We computed longitudinal field $\left(B_{l}\right)$ values and their error bars $\left(\sigma_{B_{l}}\right)$ from the LSD profiles. The error bars for Musicos and Narval data are of the order of $8 \mathrm{G}$ and $2 \mathrm{G}$, respectively. We find that the $B_{l}$ values are all compatible with 0 within $3 \sigma_{B_{l}}$. The field values and their error bars are reported in Tables 1 and 2 . The mean longitudinal field obtained from the 34 Musicos measurements is $B_{l}=-0.5 \pm 1.2 \mathrm{G}$. The mean longitudinal field obtained from the 23 Narval measurements is $B_{l}=-0.1 \pm 0.4 \mathrm{G}$. These results are not compatible with the field values proposed by BP07.

In the top panel of Figs. 4 and 5 the longitudinal field values obtained from Musicos and Narval observations, respectively, are shown folded with the ephemeris suggested by BP07. The null $N$ values are also reported in the bottom panel of each figure. A sinusoidal dipole fit of the $B_{l}$ and $N$ data is performed (black solid line) and compared to a null field (dashed line).

For Musicos data, we rejected one point for the sinusoidal fit, for which the $N$ value is higher than twice its error bar (see purple point in Fig. 4). The best sinusoidal fit for $B_{l}$ has an amplitude of $1.2 \mathrm{G}$ and is centred at $B_{0}=-0.6 \mathrm{G}$. For $N$ the best fit has an amplitude of $1.3 \mathrm{G}$ and is centred at $B_{0}=-1.3 \mathrm{G}$. We find that the reduced $\chi^{2}$ for $B_{l}$ values is $\chi^{2}=0.97$ for the dipole fit and $\chi^{2}=0.93$ for the null field. For the $N$ values it is $\chi^{2}=$ 0.96 for both the dipole fit and the null field. Therefore all four fits are acceptable (i.e. a dipole fit is not better than no field) and the $B_{l}$ values do not show a more significant sinusoidal variation than the $N$ values.

For Narval data, the best sinusoidal fit for the $B_{l}$ has an amplitude of $1.24 \mathrm{G}$ and is centred at $B_{0}=-0.12 \mathrm{G}$. For $N$ the best fit has an amplitude of $0.75 \mathrm{G}$ and is centred at $B_{0}=0.02 \mathrm{G}$. We find that the reduced $\chi^{2}$ for $B_{l}$ values is $\chi^{2}=0.77$ for the dipole fit and $\chi^{2}=1.01$ for the null field. For the $N$ values it is $\chi^{2}=0.87$ for the dipole fit and $\chi^{2}=0.83$ for the null field. Therefore we find again that all four fits are acceptable (i.e. a dipole fit is not better than no field) and the $B_{l}$ values do not show a more significant sinusoidal variation than the $N$ values.

We conclude that we cannot reproduce the sinusoidal variation with an amplitude of $20 \mathrm{G}$ centred on $B_{0}=11 \mathrm{G}$ claimed by BP07, shown in red in Figs. 4 and 5.

In Fig. 6 we show the LSD $V$ profiles and dynamical plot of these profiles folded in phase with the ephemeris of BP07 

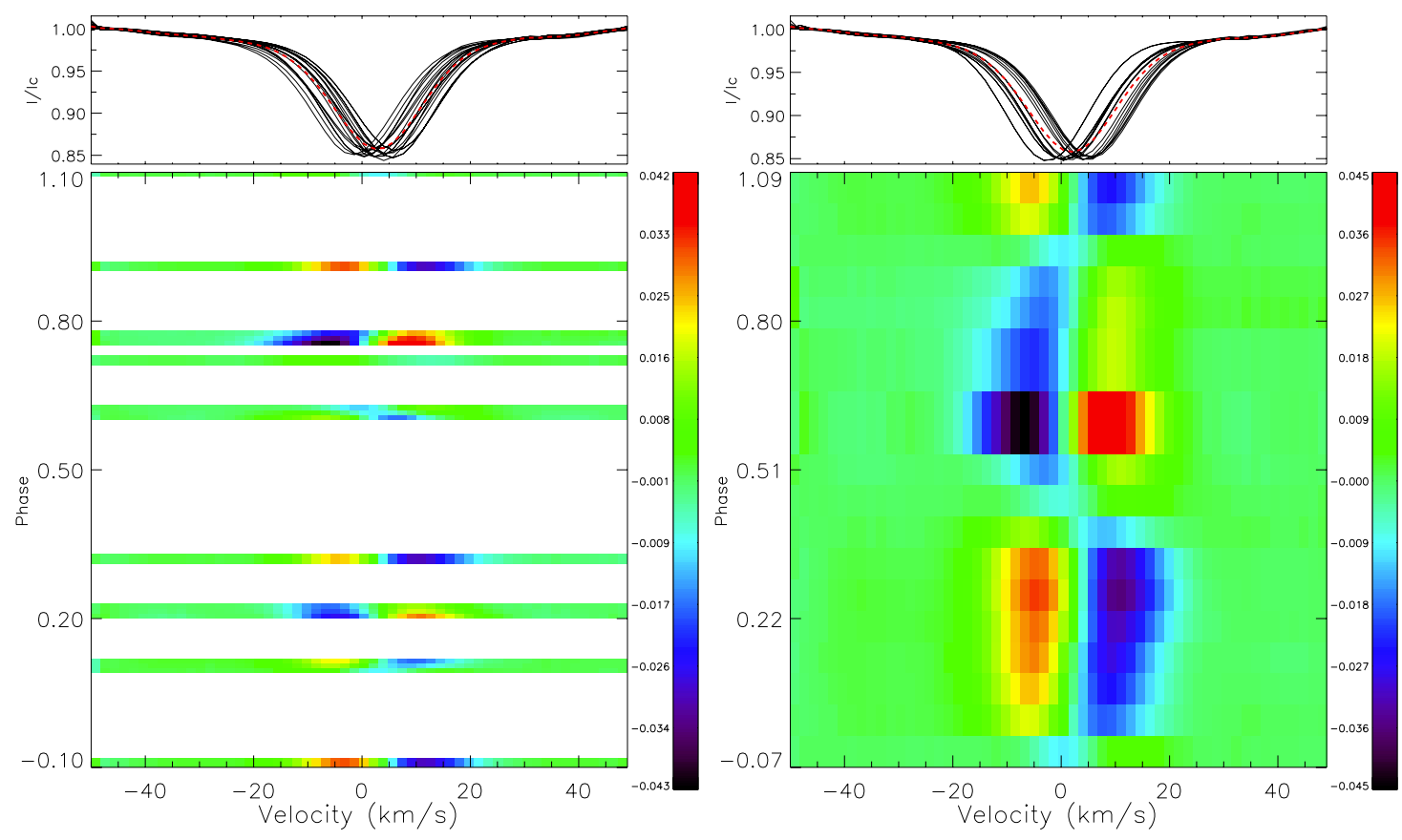

Fig. 3. Top: Narval LSD I profiles. The mean profile is shown with a dashed red line. Bottom: dynamical plot of the residuals of the LSD I profiles compared to the mean LSD I profile, folded in phase with the ephemeris of BP07 (left) and with the same $\mathrm{HJD}_{0}$ but the main pulsation period $P_{\text {puls }}=0.15175 \mathrm{~d}($ right $)$. The profiles have been rebinned in bins of 0.01 for the period of BP07 and 0.06 for the pulsation period.

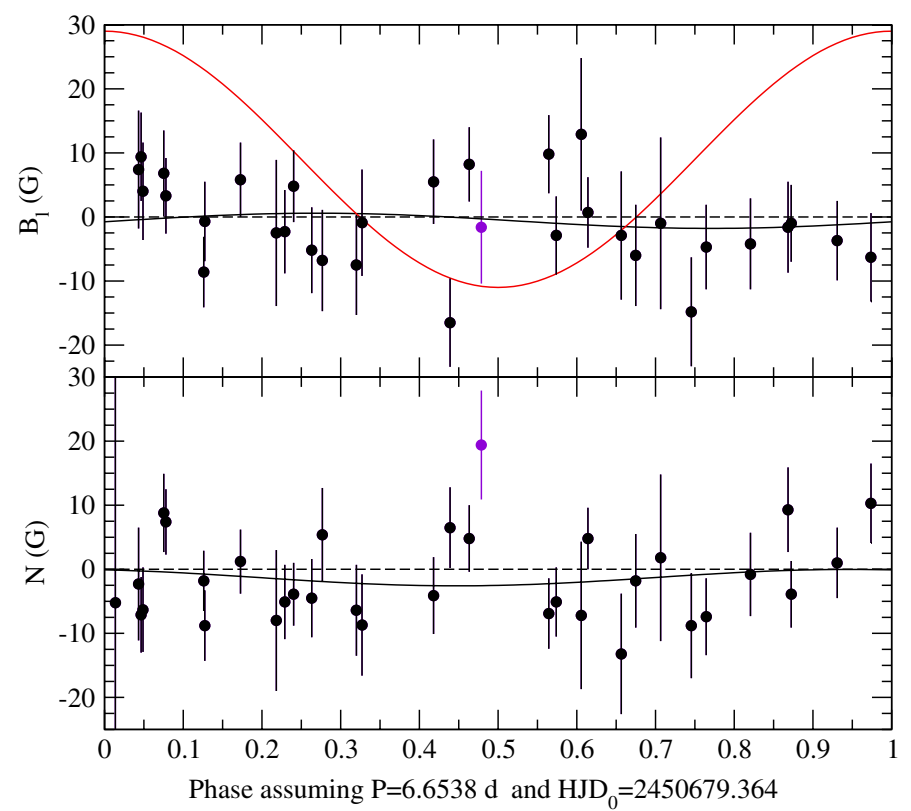

Fig. 4. Longitudinal field measurements from the LSD Stokes $V$ profiles (top) and null $N$ measurements (bottom) observed with Musicos. The black solid lines show the best sinusoidal dipole fit to the data, the dashed lines show a null field, and the red solid line shows the sinusoidal variation expected from BP07. The purple point has been discarded for the fit, due to its high $N$ value.

rebinned in bins of 0.01 . We see no signatures in the LSD $V$ profiles and no travelling features in the dynamical plot.

\subsection{Upper limit on field strength}

To derive an upper limit on the strength of a magnetic field which could have remained undetected in our data, we used the best data available, i.e. the Narval dataset.

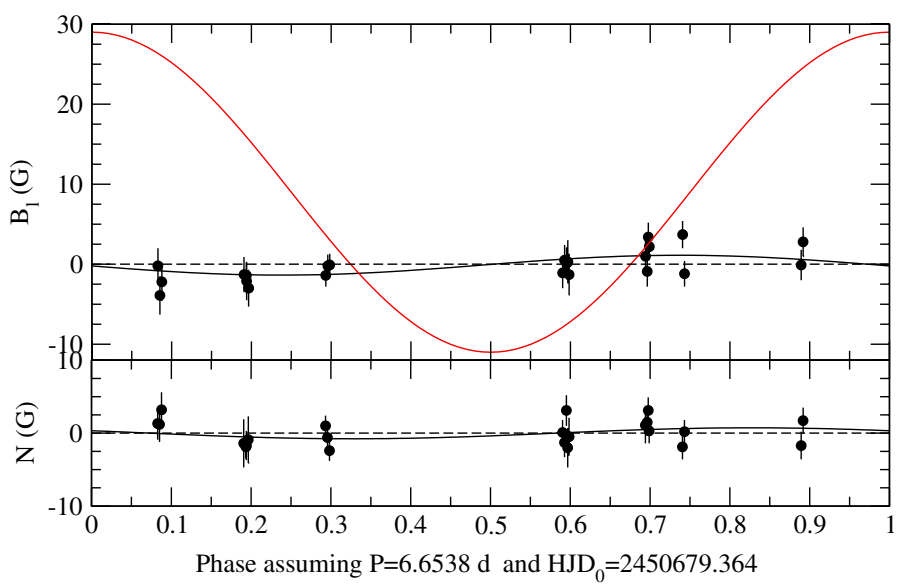

Fig. 5. Longitudinal field measurements from the LSD Stokes $V$ profiles (top) and null $N$ measurements (bottom) observed with Narval. The black solid lines show the best sinusoidal dipole fit to the data, the dashed lines show a null field, and the red solid line shows the sinusoidal variation expected from BP07.

For various values of the polar magnetic field $B_{\text {pol }}$, we calculated 1000 oblique dipole models of each of the 23 Stokes $V$ profiles with random inclination angle $\mathrm{i}$ and obliquity angle $\beta$, random rotational phase, and a white Gaussian noise with a null average and a variance corresponding to the $\mathrm{S} / \mathrm{N}$ of each Narval profile. To calculate these oblique dipole models, we used Gaussian local intensity profiles with a width calculated according to the resolving power of Narval and a thermal broadening. This broadening and the depth of the intensity profile was determined by fitting the observed LSD I profiles. We then calculated local Stokes $V$ profiles assuming the weak-field case and integrated over the visible hemisphere of the star. We obtained synthetic LSD Stokes $V$ profiles, which we normalised to the 


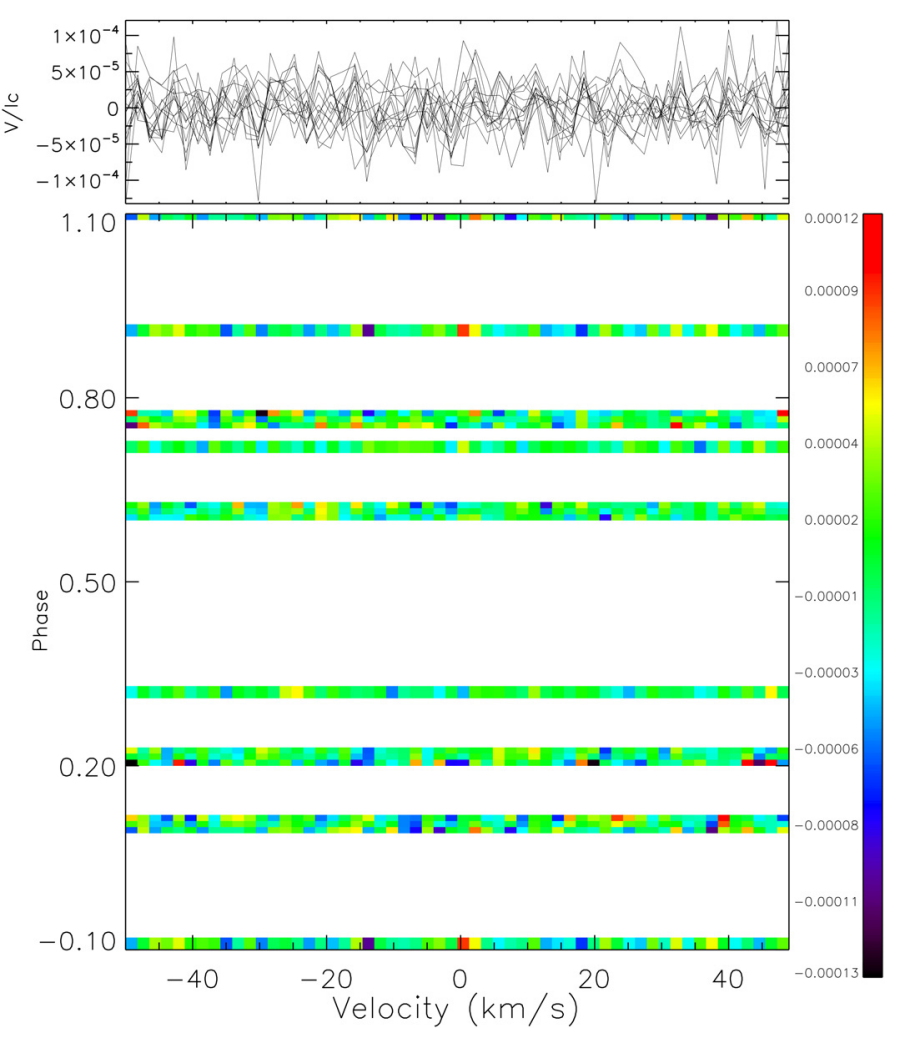

Fig. 6. Top: Narval LSD Stokes $V$ profiles. Bottom: dynamical plot of the LSD $V$ profiles, folded in phase with the ephemeris of BP07. The profiles have been rebinned in bins of 0.01 .

intensity continuum. We used the mean Landé factor and wavelength from each LSD profile.

We then computed the probability of detection of a field in this set of models by applying the Neyman-Pearson likelihood ratio test (see e.g. Helstrom 1995; Kay 1998; Levy 2008) to decide between two hypotheses, $H_{0}$ and $H_{1}$, where $H_{0}$ corresponds to noise only, and $H_{1}$ to a noisy simulated Stokes $V$ signal. This rule selects the hypothesis that maximises the probability of detection while ensuring that the probability of false alarm $P_{\mathrm{FA}}$ is not higher than a prescribed value considered acceptable. Following values usually assumed in the literature on magnetic field detections (e.g. Donati et al. 1997), we used $P_{\mathrm{FA}}=10^{-5}$ for a definite magnetic detection and $P_{\mathrm{FA}}=10^{-3}$ for a marginal magnetic detection. We then calculated the rate of detections among the 1000 models for each of the 23 profiles depending on the field strength. The definite and marginal detections in at least one of the 23 LSD profiles rate curves are plotted in Fig. 7.

This translates into an upper limit for the possible nondetected polar field strength of $B_{\mathrm{pol}}=30$ and $41 \mathrm{G}$ for a $90 \%$ chance of marginal or definite detection, respectively, in at least one of the $23 \mathrm{LSD}$ profiles. This value is $B_{\mathrm{pol}}=14$ and $19 \mathrm{G}$ for a $50 \%$ chance of marginal or definite detection, respectively. In other words, an oblique dipolar magnetic field above $\sim 40 \mathrm{G}$ at the poles should have been detected.

\section{Discussion}

\subsection{Dipolar magnetic field}

The data obtained with Narval leave no doubt that $\gamma$ Peg does not host a $B_{\mathrm{pol}}=570 \mathrm{G}$ dipolar field as suggested by BP07. This confirms the non-detection published by previous authors

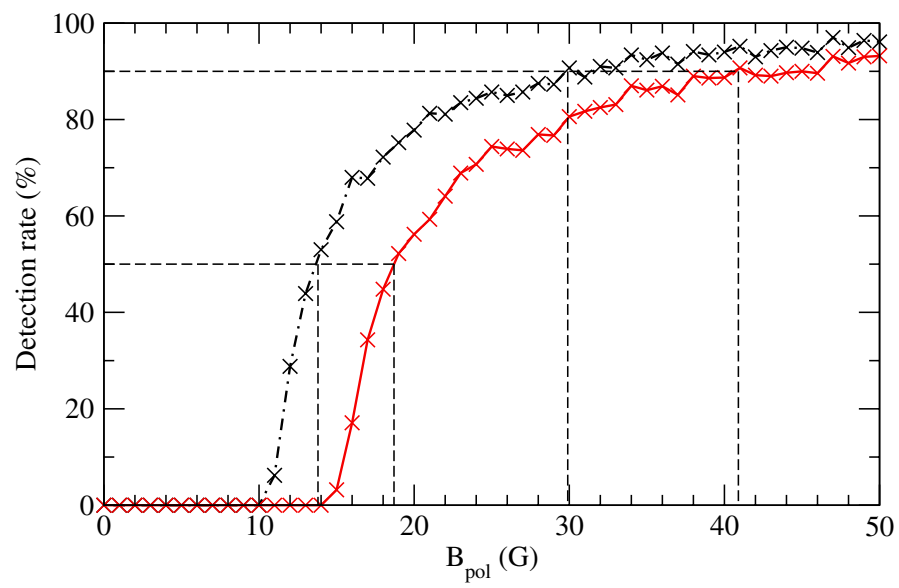

Fig. 7. Chances that an oblique dipolar magnetic field would have been detected in $\gamma$ Peg, as a definite (red solid line) or marginal (black dashed-dotted line) detection, in at least one of the 23 Narval measurements according to the strength of the dipolar magnetic field. The thin dashed lines indicate the $50 \%$ and $90 \%$ detection rate.

based on single measurements (Schnerr et al. 2008; Silvester et al. 2009) and obtained from our older series of Musicos data presented here. The mean longitudinal field value obtained from metallic lines with Narval is $B_{l}=-0.1 \pm 0.4 \mathrm{G}$ and the upper limit on the polar strength of a non-detected field is $\sim 40 \mathrm{G}$ for a $90 \%$ chance of definite detection.

In addition, although the DAO data provide longitudinal field values with higher error bars than LSD profiles extracted from Musicos and Narval data, the lower resolution of dimaPol and its fast switching device allow us to check for the presence of a magnetic field in a single broader hydrogen line. The DAO measurements of magnetic field in the $\mathrm{H} \beta$ line confirms the nondetection result.

Therefore we conclude that $\gamma$ Peg does not host the kind of stable magnetic fossil field observed in $7 \%$ of massive and intermediate-mass stars (Wade et al. 2013).

\subsection{Variability}

A period around $6.6 \mathrm{~d}$ appears in the magnetic measurements collected by BP07 and cannot be ruled out in our DAO data. Its 1 -d alias $\left(f \sim 0.85 \mathrm{c} \mathrm{d}^{-1}\right)$ lies within the range of g-mode frequencies observed in $\gamma$ Peg. Variability due to pulsations can easily appear in magnetic measurements if the exposure time is longer than about $1 / 20$ of the pulsation period. In the case of the observed g-modes, this would correspond to exposures longer than $\sim 5000 \mathrm{~s}$. BP07 do not indicate the duration of their exposures, therefore it is not possible to check whether the length of their exposures is the reason for the detection of this period in their magnetic measurements.

The much shorter and higher S/N Narval exposures do not show the variability around $6.6 \mathrm{~d}$. Some of our DAO exposures are of the order of $\sim 5000$-s duration. However, the rapid switching of the wave plate in dimaPol should avoid a pollution of the measurements by stellar variations such as pulsations.

In addition, while we are able to find a sinusoidal fit to the DAO data with BP07's period but a completely different phasing (see Fig. 1), we are also able to find similarly good sinusoidal fits with a variety of other periods (see Fig. 2). Therefore the fit to DAO data with BP07's period does not appear to be significant. 


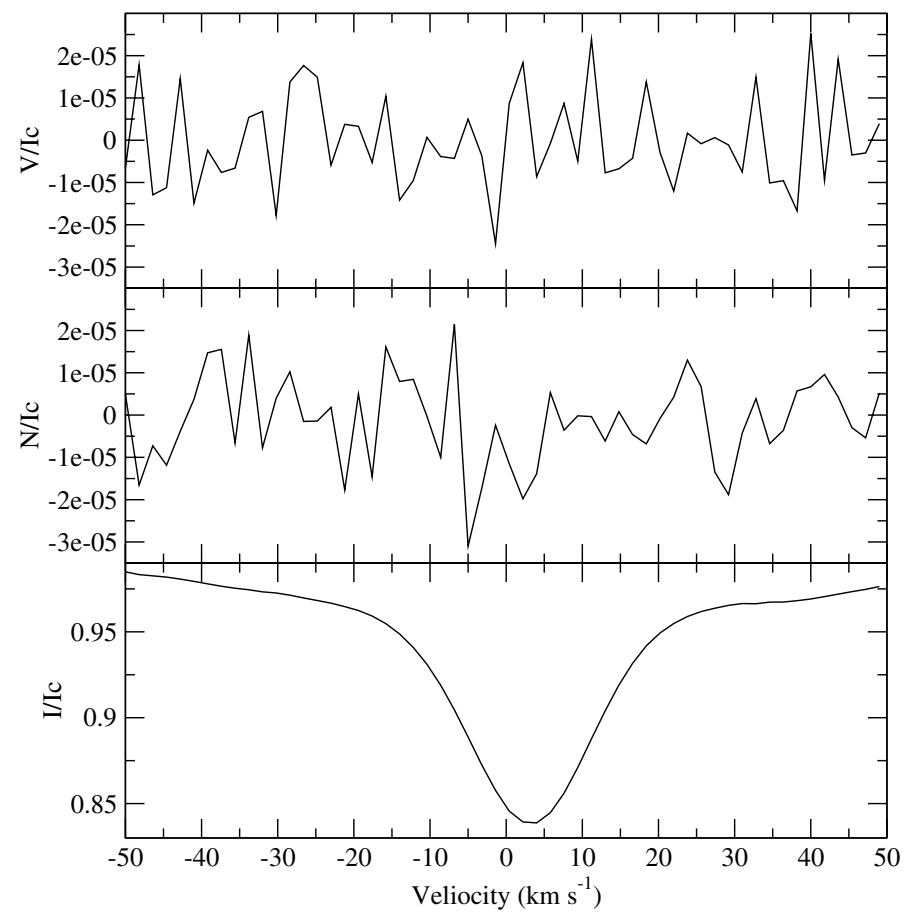

Fig. 8. Average Narval LSD Stokes $V$ (top), null polarisation (middle) and intensity (bottom) profiles.

\subsection{Vega-like magnetic field}

Very weak magnetic fields have been detected in a few A stars (e.g. Petit et al. 2011). The first such field was discovered in the star Vega (Lignières et al. 2009; Petit et al. 2010) and this is why they are called "Vega-like fields". The existence of these very weak fields in A stars is still debated and no such field has ever been detected in an $\mathrm{O}$ or B star.

For Vega, the measured longitudinal field is $-0.6 \pm 0.3 \mathrm{G}$ (Lignières et al. 2009). For Sirius, the measured Vega-like longitudinal field is $0.2 \pm 0.1 \mathrm{G}$. These measurements, although weak (a few tens of a Gauss), are not compatible with 0 . Moreover, the weak signature of these fields is observed in the average LSD Stokes $V$ profiles of these stars (see Fig. 1 in Petit et al. 2011).

For $\gamma$ Peg, with our best measurements (i.e. Narval data) and using the same method as Lignières et al. (2009) and Petit et al. (2011), we find a longitudinal field of $-0.1 \pm 0.4 \mathrm{G}$. Contrary to Vega and Sirius, this field is compatible with 0 . Moreover, we find no signature in the average LSD Stokes $V$ profile, as shown in Fig. 8. Therefore we can also exclude that the longitudinal field has been averaged out over the stellar surface.

We thus conclude that $\gamma$ Peg does not host a Vega-like field.

\subsection{On the existence of Vega-like fields in most OBA stars}

In magnetic massive stars with strong (above a few hundreds Gauss) magnetic fields, the field is thought to be of fossil origin. The primordial field evolves to an equilibrium state: a twisted torus inside the star, that appears most of the time as a simple oblique dipole field at the surface (Braithwaite \& Nordlund 2006; Duez \& Mathis 2010).

After the discovery of very weak magnetic fields in Vega and Sirius, two scenarios have been proposed to explain the existence of such weak fields. First, Aurière et al. (2007) proposed that in the case of Vega-like stars, the magnetic field has reached the equilibrium but the field amplitude was too weak to freeze a possible differential rotation. As a consequence, this differential rotation created a strong toroidal field, which became unstable because of the Tayler instability (Tayler 1973). This destroyed the stable field configuration. Second, Braithwaite \& Cantiello (2013) proposed that Vega-like stars are following the same process as the strongly magnetic ones towards a magnetic equilibrium, but, because their field is much weaker, the time necessary to reach this equilibrium is longer than the current age of the star and the field is currently still evolving. If the time to reach the equilibrium is longer that the lifetime of the star, it is called a failed fossil field. Both scenarios conclude that all massive and intermediate-mass stars should host a magnetic field: either a strong stable fossil field (as observed in $7 \%$ of the stars according to the MiMeS survey, see Wade et al. 2013) or a weak Vega-like field for all the other stars.

However, we find that $\gamma$ Peg hosts neither a strong stable magnetic field nor a weak field similar to Vega or Sirius. This raises questions about the above scenarios. In particular, in order to work, both scenarios require an initial seed field, coming from the molecular cloud from which the star was formed. If the star did not capture a field during its collapse, no dynamo can develop during the pre-main sequence convective phase and there will be no relaxation towards an equilibrium fossil field in the radiative envelope later on. One can then wonder why the few A stars observed with deep spectropolarimetry so far did show a Vega-like field and thus had a seed field, and the B star $\gamma$ Peg does not. If $\gamma$ Peg did capture a seed field, it seems to have been destroyed, which cannot be explained by the above two scenarios.

To test the Vega-like field scenarios further, it would be interesting to study more bright B stars with very deep spectropolarimetry. Moreover, the A stars for which a Vega-like field was discovered so far are rather peculiar objects: Vega is a very rapid rotator seen pole-on and a pulsator, and Sirius is an Am star and a binary. It would be interesting to check for the presence of Vega-like fields in "normal" A stars.

\subsection{Implication for stellar evolution models}

The non-detection of a magnetic field in the early B star $\gamma$ Peg with a very low detection threshold implies that current stellar evolution models without magnetic fields might be a good approximation of most massive stars (Meynet \& Maeder 2000; Maeder \& Meynet 2000). A very weak field, such as a Vega-like field, could make a great difference in the evolution of a massive star and it was thus important to check whether these fields are indeed present in all massive and intermediate-mass stars. Although our work is only based on one star, we have shown that the predictions by the Vega-like field theories are too crude and there is no need to include these weak fields in all evolution models of massive stars.

\section{Conclusions}

We have performed extremely sensitive magnetic measurements of the early B star $\gamma$ Peg. We found that it does not host the several hundreds Gauss field claimed by BP07. It also does not host a very weak Vega-like magnetic field.

Our results show that, while Vega-like fields may exist in A stars, their existence in hotter stars and in most OBA stars can be questioned. Very deep magnetic observations of other bright OBA stars would allow us to test further and constrain the Vega-like theories for massive stars. 
Acknowledgements. We thank the Musicos observers H. Henrichs, V. Geers and N. Boudin, the Narval observers J. Gutiérrez-Soto and F. Cochard, as well as the TBL service observation team. C.N. wishes to thank the Programme National de Physique Stellaire (PNPS) for their support and acknowledges support from the ANR (Agence Nationale de la Recherche) project Imagine. C.N. thanks G. Meynet for fruitful discussions, which led to the Narval observations presented here. This research has made use of the SIMBAD database operated at CDS, Strasbourg (France), and of NASA's Astrophysics Data System (ADS).

\section{References}

Aurière, M., Wade, G. A., Silvester, J., et al. 2007, A\&A, 475, 1053

Braithwaite, J., \& Cantiello, M. 2013, MNRAS, 428, 2789

Braithwaite, J., \& Nordlund, A. 2006, A\&A, 450, 1077

Briquet, M., Neiner, C., Aerts, C., et al. 2012, MNRAS, 427, 483

Butkovskaya, V. V., \& Plachinda, S. I. 2007, A\&A, 469, 1069

Chapellier, E., Le Contel, D., Le Contel, J. M., Mathias, P., \& Valtier, J.-C. 2006, A\&A, 448, 697

Donati, J.-F., Catala, C., Wade, G. A., et al. 1999, A\&AS, 134, 149

Donati, J.-F., Semel, M., Carter, B. D., Rees, D. E., \& Collier Cameron, A. 1997, MNRAS, 291, 658

Duez, V., \& Mathis, S. 2010, A\&A, 517, A58

Handler, G., Matthews, J. M., Eaton, J. A., et al. 2009, ApJ, 698, L56

Heger, A., Woosley, S. E., \& Spruit, H. C. 2005, ApJ, 626, 350

Helstrom, C. W. 1995, Elements of Signal Detection and Estimation (Prentice Hall)

Kay, S. M. 1998, Fundamentals of Statistical Signal Processing, Volume 2: Detection Theory (Prentice Hall)

Kupka, F., Piskunov, N., Ryabchikova, T. A., Stempels, H. C., \& Weiss, W. W. 1999, A\&AS, 138, 119
Landstreet, J. D. 1992, A\&ARv., 4, 35

Levy, B. C. 2008, Principles of Signal Detection and Parameter Estimation (Springer)

Lignières, F., Petit, P., Böhm, T., \& Aurière, M. 2009, A\&A, 500, L41

Maeder, A., \& Meynet, G. 2000, ARA\&A, 38, 143

Maeder, A., \& Meynet, G. 2003, A\&A, 411, 543

Mathis, S., \& Zahn, J.-P. 2005, A\&A, 440, 653

McAlister, H. A., Hartkopf, W. I., Sowell, J. R., Dombrowski, E. G., \& Franz, O. G. 1989, AJ, 97, 510

Meynet, G., \& Maeder, A. 2000, A\&A, 361, 101

Monin, D., Bohlender, D., Hardy, T., Saddlemyer, L., \& Fletcher, M. 2012, PASP, 124, 329

Morel, T., Hubrig, S., \& Briquet, M. 2008, A\&A, 481, 453

Petit, P., Lignières, F., Wade, G. A., et al. 2010, A\&A, 523, A41

Petit, P., Lignières, F., Aurière, M., et al. 2011, A\&A, 532, L13

Piskunov, N. E., Kupka, F., Ryabchikova, T. A., Weiss, W. W., \& Jeffery, C. S. 1995, A\&AS, 112, 525

Roberts, Jr., L. C., Turner, N. H., \& ten Brummelaar, T. A. 2007, AJ, 133, 545

Schnerr, R. S., Henrichs, H. F., Neiner, C., et al. 2008, A\&A, 483, 857

Silvester, J., Neiner, C., Henrichs, H. F., et al. 2009, MNRAS, 398, 1505

Spruit, H. C. 1999, A\&A, 349, 189

Tayler, R. J. 1973, MNRAS, 161, 365

Telting, J. H., Schrijvers, C., Ilyin, I. V., et al. 2006, A\&A, 452, 945

Wade, G. A., Grunhut, J., Alecian, E., et al. 2013, in Magnetic fields throughout stellar evolution, Proc. IAU Symp., 302 [arXiv: 1310. 3965]

Walczak, P., \& Daszyńska-Daszkiewicz, J. 2010, Astron. Nachr., 331, 1057

Zahn, J.-P. 2011, in IAU Symp. 272, eds. C. Neiner, G. Wade, G. Meynet, \& G. Peters, 14

Zahn, J.-P., Brun, A. S., \& Mathis, S. 2007, A\&A, 474, 145 
Table 1. Journal of 34 Musicos observations of $\gamma$ Peg.

\begin{tabular}{llllrrr}
\hline \hline$\#$ & Date & Mid-HJD & $T_{\text {exp }}$ & $S / N$ & $B_{l}$ & $\sigma_{B_{l}}$ \\
& & -2450000 & s & & $\mathrm{G}$ & $\mathrm{G}$ \\
\hline 1 & 19dec.01 & 2263.27782 & $4 \times 300$ & 950 & 9.4 & 6.9 \\
2 & 19dec.01 & 2263.29387 & $4 \times 300$ & 840 & 4.0 & 7.6 \\
3 & 20jun.02 & 2446.65110 & $4 \times 180$ & 460 & 12.9 & 11.9 \\
4 & 27jun.02 & 2453.64276 & $4 \times 180$ & 580 & -2.9 & 10.0 \\
5 & 15nov.04 & 3325.41329 & $4 \times 360$ & 730 & -6.0 & 7.9 \\
6 & 16nov.04 & 3326.38272 & $4 \times 600$ & 860 & -4.2 & 7.1 \\
7 & 17nov.04 & 3327.40198 & $4 \times 600$ & 880 & -6.3 & 6.9 \\
8 & 18nov.04 & 3328.42364 & $4 \times 600$ & 990 & -0.7 & 6.2 \\
9 & 20nov.04 & 3330.35798 & $4 \times 600$ & 920 & 5.5 & 6.6 \\
10 & 21nov.04 & 3331.39483 & $4 \times 600$ & 1030 & -2.9 & 6.1 \\
11 & 22nov.04 & 3332.27740 & $4 \times 600$ & 410 & -1.0 & 13.4 \\
12 & 23nov.04 & 3333.38135 & $4 \times 600$ & 1060 & -1.0 & 6.0 \\
13 & 24nov.04 & 3334.32173 & $4 \times 600$ & 410 & -38.2 & 47.2 \\
14 & 25nov.04 & 3335.37968 & $4 \times 600$ & 1120 & 5.8 & 5.8 \\
15 & 26nov.04 & 3336.35965 & $4 \times 600$ & 770 & -7.5 & 7.8 \\
16 & 27nov.04 & 3337.41602 & $4 \times 600$ & 680 & -1.6 & 8.8 \\
17 & 30nov.04 & 3340.42430 & $4 \times 600$ & 1000 & -3.7 & 6.2 \\
18 & 02jul.05 & 3554.64367 & $4 \times 300$ & 1160 & -8.6 & 5.5 \\
19 & 03jul.05 & 3555.64737 & $4 \times 300$ & 750 & -6.8 & 7.9 \\
20 & 10jul.05 & 3562.63569 & $4 \times 300$ & 680 & -0.9 & 8.3 \\
21 & 15jul.05 & 3567.61350 & $4 \times 300$ & 910 & 6.8 & 6.7 \\
22 & 15jul.05 & 3567.63220 & $4 \times 400$ & 1080 & 3.3 & 5.9 \\
23 & 16jul.05 & 3568.56584 & $4 \times 300$ & 490 & -2.5 & 11.4 \\
24 & 16jul.05 & 3568.63673 & $4 \times 400$ & 910 & -2.3 & 6.5 \\
25 & 03dec.05 & 3708.44124 & $4 \times 900$ & 1160 & 4.8 & 5.6 \\
26 & 10dec.05 & 3715.24809 & $4 \times 300$ & 890 & -5.2 & 6.7 \\
27 & 11dec.05 & 3716.41796 & $4 \times 300$ & 860 & -16.5 & 6.9 \\
28 & 12dec.05 & 3717.25230 & $4 \times 300$ & 990 & 9.8 & 6.1 \\
29 & 13dec.05 & 3718.45764 & $4 \times 300$ & 730 & -14.8 & 8.5 \\
30 & 14dec.05 & 3719.27452 & $4 \times 300$ & 830 & -1.6 & 7.1 \\
31 & 15dec.05 & 3720.43865 & $4 \times 300$ & 620 & 7.4 & 9.2 \\
32 & 18dec.05 & 3723.23341 & $4 \times 300$ & 1100 & 8.2 & 5.8 \\
33 & 19dec.05 & 3724.23705 & $4 \times 300$ & 1200 & 0.7 & 5.5 \\
34 & 20dec.05 & 3725.23694 & $4 \times 300$ & 1010 & -4.7 & 6.6 \\
\hline & & & & & & \\
\hline
\end{tabular}

Notes. The $\mathrm{S} / \mathrm{N}$ given in Col. 5 is the one measured at $5000 \AA$ in the I spectrum.
Table 2. Journal of 23 Narval observations of $\gamma$ Peg.

\begin{tabular}{|c|c|c|c|c|c|c|c|}
\hline \# & $\begin{array}{l}\text { Date } \\
2007\end{array}$ & $\begin{array}{l}\text { Mid-HJD } \\
-2450000\end{array}$ & $\begin{array}{l}T_{\text {exp }} \\
\mathrm{s}\end{array}$ & $S / N$ & Lines & $\begin{array}{l}B_{l} \\
\mathrm{G}\end{array}$ & $\begin{array}{r}\sigma_{B_{l}} \\
\mathrm{G} \\
\end{array}$ \\
\hline 1 & 18nov. & 4423.41795 & $4 \times 300$ & 1680 & 744 & 1.0 & 2.4 \\
\hline 2 & 18nov. & 4423.43313 & $4 \times 120$ & 990 & 1012 & -0.9 & 1.9 \\
\hline 3 & 18nov. & 4423.44134 & $4 \times 120$ & 1060 & 1012 & 3.4 & 1.8 \\
\hline 4 & 18 nov. & 4423.44951 & $4 \times 120$ & 1070 & 1012 & 2.2 & 1.7 \\
\hline 5 & 24 nov. & 4429.42376 & $4 \times 120$ & 660 & 1012 & 0.3 & 2.7 \\
\hline 6 & 24 nov. & 4429.43503 & $4 \times 200$ & 690 & 1012 & -1.3 & 2.6 \\
\hline 7 & 28nov. & 4433.39564 & $4 \times 200$ & 1130 & 1012 & -1.4 & 1.6 \\
\hline 8 & 04dec. & 4439.31207 & $4 \times 300$ & 790 & 1012 & -0.2 & 2.2 \\
\hline 9 & 04dec. & 4436 & $4 \times 300$ & 730 & 101 & -3.9 & 2.4 \\
\hline 10 & 04dec. & 62 & $4 \times$ & 740 & 101 & -2.2 & 2.4 \\
\hline 11 & $12 \mathrm{dec}$. & 42 & $4 \times$ & 1260 & 1012 & -1.4 & 1.4 \\
\hline 12 & $12 \mathrm{dec}$. & 4447 & $4 \times$ & 1320 & 979 & -0.2 & 1.4 \\
\hline 13 & $12 \mathrm{dec}$. & 4447.39898 & $4 \times 300$ & 1340 & 950 & -0.1 & 1.4 \\
\hline 14 & 14dec. & 4449.34232 & $4 \times 300$ & 1590 & 791 & -1.1 & 1.9 \\
\hline 15 & 14dec. & 4449.35861 & $4 \times 300$ & 1560 & 808 & 0.5 & 1.9 \\
\hline 16 & 14dec. & 4449.37489 & $4 \times 300$ & 1480 & 864 & 0.1 & 2.0 \\
\hline 17 & $15 \mathrm{dec}$. & 4450.34251 & $4 \times 300$ & 1070 & 1012 & 3.7 & 1.7 \\
\hline 18 & $15 \mathrm{dec}$. & 4450.35880 & $4 \times 300$ & 1140 & 1012 & -1.2 & 1.6 \\
\hline 19 & 16dec. & 4451.33234 & $4 \times 300$ & 950 & 1012 & -0.1 & 1.9 \\
\hline 20 & 16dec. & 4451.34861 & $4 \times 300$ & 1000 & 1012 & 2.8 & 1.8 \\
\hline 21 & 18dec. & 4453.33791 & $4 \times 300$ & 1640 & 794 & -1.3 & 2.2 \\
\hline 22 & 18dec. & 4453.35963 & $4 \times 300$ & 1660 & 758 & -2.1 & 2.4 \\
\hline 23 & 18dec. & 4453.37592 & $4 \times 300$ & 1520 & 848 & -3.0 & 2.3 \\
\hline
\end{tabular}

Notes. The $\mathrm{S} / \mathrm{N}$ given in col. 5 is the one measured at $5000 \AA$ in the I spectrum. Column 6 indicates the number of lines used in the LSD mask.

Table 3. Journal of 18 DAO observations of $\gamma$ Peg.

\begin{tabular}{|c|c|c|c|c|c|c|}
\hline \# & Date & $\begin{array}{l}\text { Mid-HJD } \\
-2450000\end{array}$ & $\begin{array}{l}T_{\exp } \\
\mathrm{s}\end{array}$ & $\mathrm{S} / \mathrm{s} . S / N$ & & $\begin{array}{l}\sigma_{B_{l}} \\
\mathrm{G}\end{array}$ \\
\hline 1 & 27 dec. 07 & 4461.65079 & $40 \times 30=1200$ & 301820 & -41 & 38 \\
\hline 2 & $27 \mathrm{dec} .07$ & 4461.66969 & $80 \times 15=1200$ & 301820 & -13 & 47 \\
\hline 3 & $27 \mathrm{dec} .07$ & 4461.68948 & $40 \times 30=1200$ & 21620 & 62 & 75 \\
\hline 4 & 27dec.07 & 4461.70234 & $20 \times 30=630$ & 61100 & 53 & 87 \\
\hline 5 & 27 dec. 07 & 4461.71476 & $20 \times 30=600$ & 101020 & 77 & 87 \\
\hline 6 & 7 sep. 08 & 4716.89516 & $30 \times 30=900$ & 601610 & 84 & 67 \\
\hline 7 & 8sep.08 & 4717.90738 & $19 \times 18=342$ & 601240 & -34 & 64 \\
\hline 8 & 8sep.08 & 4717.91400 & $20 \times 18=360$ & 101270 & 14 & 79 \\
\hline 9 & 8sep.08 & 4717.92539 & $20 \times 18=360$ & 601260 & -8 & 57 \\
\hline 10 & $2 \mathrm{dec} .09$ & 5167.70960 & $60 \times 30=1800$ & 601730 & 10 & 57 \\
\hline 11 & 3dec.09 & 5168.69853 & $79 \times 30=2370$ & 602220 & 45 & 38 \\
\hline 12 & 4dec.09 & 5169.67541 & $100 \times 30=3000$ & 602380 & 28 & 37 \\
\hline 13 & 5 dec. 09 & 5170.64841 & $80 \times 60=4800$ & 602090 & -6 & 44 \\
\hline 14 & 6dec.09 & 5171.64258 & $94 \times 30=2820$ & 601890 & -38 & 53 \\
\hline 15 & 26 dec. 09 & 5191.59017 & $100 \times 30=3000$ & 601780 & 60 & 48 \\
\hline 16 & $27 \mathrm{dec} .09$ & 5192.71002 & $100 \times 30=3000$ & 601370 & 103 & 62 \\
\hline 17 & 28 dec. 09 & 5193.66309 & $100 \times 30=3000$ & 601670 & 102 & 50 \\
\hline 18 & 3 jan. 10 & 5199.64027 & $99 \times 30=2970$ & 601550 & 66 & 69 \\
\hline
\end{tabular}

Notes. The reported field values have been measured in the $\mathrm{H} \beta$ line. Column 4 provides the number of sub-exposures, individual exposure time as well as total exposure time for each measurement. Column 5 indicates the number of times the plate has been switched per sub-exposure. 